\title{
Regulatory interactions between long noncoding RNA LINC00968 and miR-9-3p in non-small cell lung cancer: A bioinformatic analysis based on miRNA microarray, GEO and TCGA
}

\author{
DONG-YAO LI ${ }^{1}$, WEN-JIE CHEN ${ }^{1}$, JUN SHANG ${ }^{1}$, GANG CHEN $^{2}$ and SHI-KANG LI ${ }^{1}$ \\ Departments of ${ }^{1}$ Thoracic and Cardiovascular Surgery, and ${ }^{2}$ Pathology, First Affiliated Hospital of Guangxi \\ Medical University, Nanning, Guangxi Zhuang Autonomous Region 530021, P.R. China
}

Received October 13, 2017; Accepted February 28, 2018

DOI: $10.3892 / \mathrm{ol} .2018 .8476$

\begin{abstract}
Long non-coding RNAs (lncRNAs) have been demonstrated to mediate carcinogenesis in various types of cancer. However, the regulatory role of lncRNA LINC00968 in lung adenocarcinoma remains unclear. The microRNA (miRNA) expression in LINC00968-overexpressing human lung adenocarcinoma A549 cells was detected using miRNA microarray analysis. miR-9-3p was selected for further analysis, and its expression was verified in the Gene Expression Omnibus (GEO) database. In addition, the regulatory axis of LINC00968 was validated using The Cancer Genome Atlas (TCGA) database. Results of the GEO database indicated miR-9-3p expression in lung adenocarcinoma was significantly higher compared with normal tissues. Functional enrichment analyses of the target genes of miR-9-3p indicated protein binding and the AMP-activated protein kinase pathway were the most enriched Gene Ontology and KEGG terms, respectively. Combining target genes with the correlated genes of LINC00968 and miR-9-3p, 120 objective genes were obtained, which were used to construct a protein-protein interaction (PPI) network. Cyclin A2 (CCNA2) was identified to have a vital role in the PPI network. Significant correlations were detected between LINC00968, miR-9-3p and CCNA2 in lung adenocarcinoma. The LINC00968/miR-9-3p/CCNA2 regulatory axis provides a new foundation for further evaluating the
\end{abstract}

Correspondence to: Dr Shi-Kang Li, Department of Thoracic and Cardiovascular Surgery, First Affiliated Hospital of Guangxi Medical University, 6 Shuangyong Road, Nanning, Guangxi Zhuang Autonomous Region 530021, P.R. China

E-mail: shikangli@hotmail.com

Dr Gang Chen, Department of Pathology, First Affiliated Hospital of Guangxi Medical University, 6 Shuangyong Road, Nanning, Guangxi Zhuang Autonomous Region 530021, P.R. China

E-mail: chen_gang_triones@163.com

Key words: LINC00968, miR-9-3p, non-small cell lung cancer, miRNA microarray, gene expression Omnibus, The Cancer Genome Atlas regulatory mechanisms of LINC00968 in lung adenocarcinoma.

\section{Introduction}

Lung cancer is responsible for $\sim 26 \%$ of all cancer mortalities worldwide (1). Despite the continuous increase in survival rates for most types of cancer, the 5-year survival rate of lung cancer has remained low, at only $18 \%$ (1). Lung cancer can be divided pathologically into two types. Most lung cancer cases (>80\%) correspond to non-small cell lung cancer (NSCLC), with the remainder being small-cell lung cancer (2). The pathogenesis of NSCLC is not fully understood, although studies about the molecular mechanisms of lung cancer continue to provide new insights into the disease.

Long noncoding RNAs (lncRNAs) are attracting increasing attention for their potential roles in numerous cancer types $(3,4)$. For example, in NSCLC, two lncRNAs-plasmacytoma variant translocation 1 (PVT1) and urothelial carcinoma-associated 1 (UCA1)-were found to promote oncogenesis and tumor growth, respectively $(5,6)$. LncRNAs may participate in tumorigenesis through the competitive endogenous RNA (ceRNA) network $(7,8)$ by interacting with microRNAs (miRNAs) (9). Differential miRNA expression has been detected in precancerous and cancerous tissues $(10,11)$. Therefore, it is possible that the ceRNA network might be important in carcinogenesis and cancer development. Furthermore, knowledge about ceRNA interactions might provide insights into the potential mechanisms and biological functions of lncRNAs in cancer. One study demonstrated that the carcinogen $\mathrm{H} 19$ acted as a ceRNA to alter expression levels of certain miRNAs (miR-138 and miR-200a) and genes [zinc finger E-box binding homeobox 1 (ZEB)1/ZEB2] in colorectal cancer (8). In addition, ceRNA networks have been identified in other cancer types, including hepatocellular, breast, pancreatic and gastric cancer (12-15).

Using bioinformatic analysis, it was previously demonstrated by the authors that the IncRNA LINC00968 was differentially expressed between normal lung and tumor tissues (16). In the present study, microarray analysis was employed to identify which miRNAs were differentially expressed with LINC00968 overexpression. Among the five identified differentially expressed 
miRNAs, miR-9-3p was selected for further analysis. In total, nine prediction algorithms were applied to identify target genes of miR-9-3p, which were then subjected to functional enrichment analysis (17-20). Finally, the lncRNA-miRNA-mRNA regulatory axis of LINC00968 was validated by bioinformatic and correlation analyses.

\section{Materials and methods}

miRNA microarray profiling of NSCLC cells. The NSCLC A549 cell line was obtained from the Cell Bank of the Chinese Academy of Sciences (Shanghai, China). A549 cells are adenocarcinoma human alveolar basal epithelial cells, maintained in F-12 culture medium (Gibco; Thermo Fisher Scientific, Inc., Waltham, MA, USA), trypsin-EDTA solution (Gibco; Thermo Fisher Scientific, Inc.) and D-Hank's solution (Shanghai GenePharma Co., Ltd., Shanghai, China). A549 cells were transduced with lentiviruses encoding LINC00968 for 24-72 h, then transduction efficiency was examined by an inverted fluorescent microscope and subsequent experimentation was performed. Considering the effects of green fluorescent protein (GFP)-containing lentiviral transfection on subsequent experiments such as apoptosis detection, a GFP-free lentivirus was selected. Optimal transfection conditions (achieving 95\% efficiency) were when the lentivirus titer reached $1 \times 10^{8} \mathrm{TU} / \mathrm{ml}$, diluted to 1:5. miRNA profiles were determined by using miRCURY LNA Array (version 18.0; Exiqon, Inc., Woburn, MA, USA) on an Axon GenePix 4000B microarray scanner (Axon Instruments; Molecular Devices, LLC, Sunnyvale, CA, USA). Results of the microarray analysis indicated significant downregulation of miR-9-3p ( $\log 2$ Ifold change $(\mathrm{FC}) \mid>0.585$, $\mathrm{P}<0.05)$.

Comparison with Gene Expression Omnibus (GEO) dataset. Data for miR-9-3p were searched and assembled in the GEO database (https://www.ncbi.nlm.nih.gov/gds/; date accessed: 4th June 2017), using the following search terms: (Lung OR pulmonary OR respiratory OR bronchi OR bronchioles OR alveoli OR pneumocytes OR 'air way') AND (cancer OR carcinoma OR tumor OR neoplas* OR malignan* OR adenocarcinoma) AND (microRNA OR miRNA OR 'micro RNA' OR 'small temporal RNA' OR 'noncoding RNA' OR ncRNA OR 'small RNA'). For microarrays, the following inclusion criteria were used: i) Lung adenocarcinoma and adjacent noncancerous tissues (or normal lung tissues) were included in each dataset; ii) sample organism was Homo sapiens; and iii) expression levels of miR-9-3p (hsa-miR-9* or hsa-miR-9-3p) in the experimental and control groups were provided or could be calculated.

The mean and standard deviations of expression values were extracted to estimate miR-9-3p levels in the test and control groups using STATA (version 12.0; StataCorp LP, College Station, TX, USA). $\mathrm{P}<0.05$ or $\mathrm{I}^{2}$ value $>50 \%$ indicated significant heterogeneity. Standard mean differences (SMDs) with 95\% confidence intervals (CIs) were used to evaluate continuous outcomes. Funnel plots were generated to evaluate publication bias. $\mathrm{P}<0.05$ was considered to indicate statistical significance.

Bioinformatic prediction of miRNA targets and functional enrichment analysis. The putative target genes of miR-9-3p were predicted using nine miRNA prediction algorithms: TargetScan (http://www.targetscan.org/), miRDB (http://www.mirdb.org/), RNA22 (https://cm.Jefferson. edu/rna22/), miRMap, mirTarBase (http://mirtarbase.mbc.nctu. edu.tw/), DIANA-microT (http://diana.imis.athena-innovation. gr/DianaTools/index.php?r=microT_CDS/iinde), GeneCards (http://www.genecards.org), TarBase (http://diana.imis.athenainnovation.gr/DianaTools/index.php? $\mathrm{r}=$ tarbase/index) and TargetMiner (https://www.isical.ac.in/ bioinfo_miu/final_ html_targetminer/hsa-miR-9-3p.html). Only target genes found in at least three databases were considered for further analysis. To clarify potential roles of the target genes, the Database for Annotation, Visualization, and Integrated Discovery (DAVID; http://david.abcc.ncifcrf.gov/) was used to conduct Gene Ontology (GO) and Kyoto Encyclopedia of Genes and Genomes (KEGG) pathway analyses. The method used to identify significant regulatory functions and enrichment pathways was based on the P-value as previously described (21).

Bioinformatic analysis of the association between LINC00968 and miR-9-3p using The Cancer Genome Atlas (TCGA) database. Level 3 RNAseq and miRNAseq data of lung adenocarcinoma were downloaded from TCGA (http://cancergenome.nih.gov/). An R package was used to distinguish differentially expressed RNAs and miRNAs between lung cancer and adjacent non-tumor lung tissues. For all P-values, the false discovery rate (FDR) was applied to correct for multiple hypothesis testing. The threshold of $\log 2 \mid \mathrm{FCl} \geq 1$ and $\mathrm{FDR}<0.05$ was considered significant. Genes with an absolute correlation coefficient $>0.1$ were regarded as correlated genes.

Construction of protein-protein interaction (PPI) network. Next, how closely connected the predicted target genes were with LINC00968 and miR-9-3p was determined. The predicted genes were integrated with the correlated genes to obtain prospective objective genes. To analyze interactions of the objective genes, the Search Tool for the Retrieval of Interacting Genes (STRING, http://string-db.org) was used to construct the PPI network (22), using a medium CI of 0.4 as a threshold.

Statistical analysis. The SPSS (version 22.0; SPSS Inc., Chicago, IL, USA) and R (version 3.4.0) (23) software packages were used to analyze all experimental data. Begg's test and Egger's test were used to detect publication bias in meta-analysis. Receiver operating characteristic (ROC) curves were constructed to evaluate the sensitivity and specificity of the molecular biological indicator in diagnostic tests. Differences in overall survival between two groups were estimated and compared using the Kaplan-Meier method with the log-rank test. FDR was used to correct the P-value for multiple testing. Pearson's correlation analysis was used to identify the association between LINC00968, miR-9-3p and CCNA2 in TCGA database. $\mathrm{P}<0.05$ was considered to indicate a statistically significant difference.

\section{Results}

Downregulation of miR-9-3p expression in LINC00968overexpressing A549 cells. The miRNAs that were 
A

Study

ID

SMD $(95 \% \mathrm{Cl}) \quad$ Weight

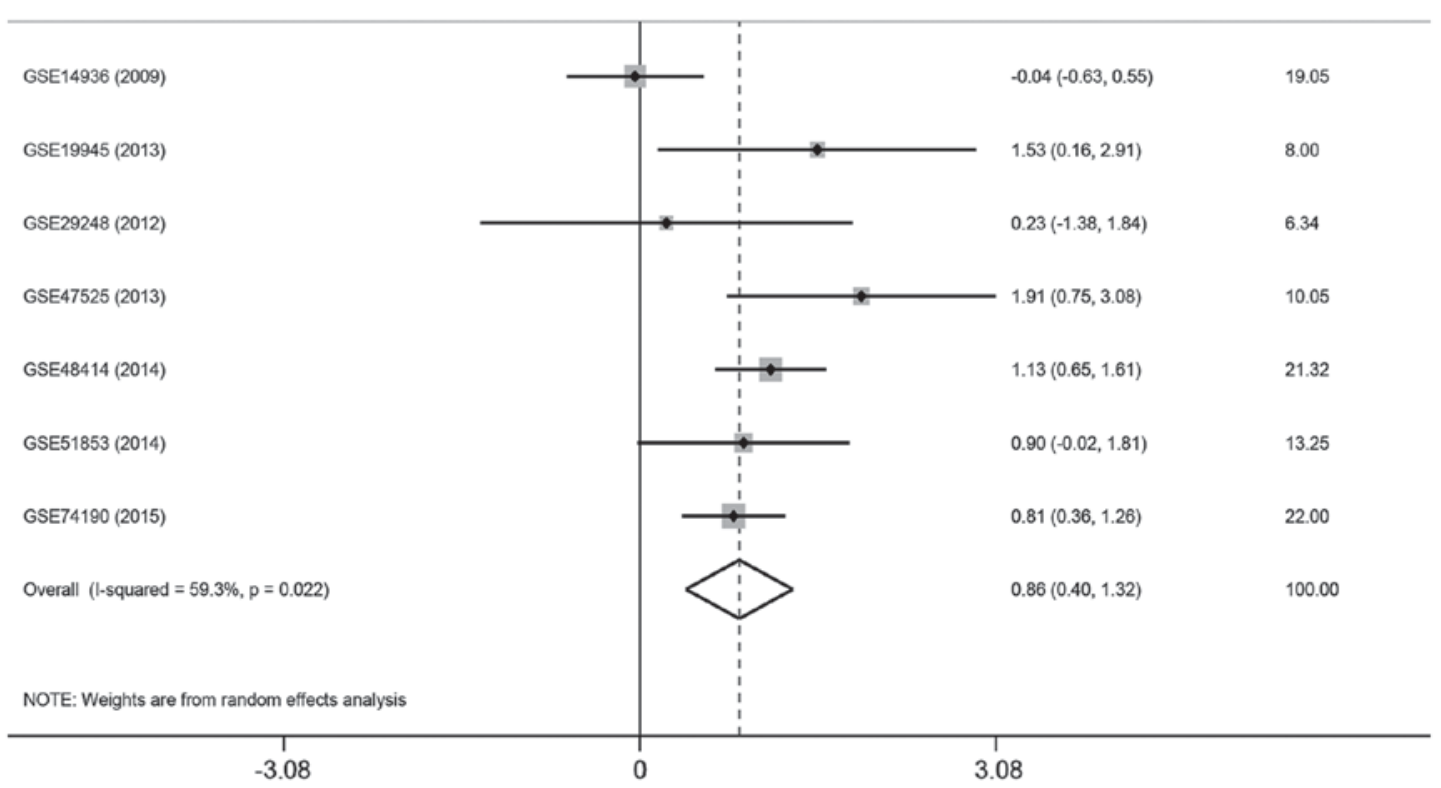

B

Begg's funnel plot with pseudo $95 \%$ confidence limits

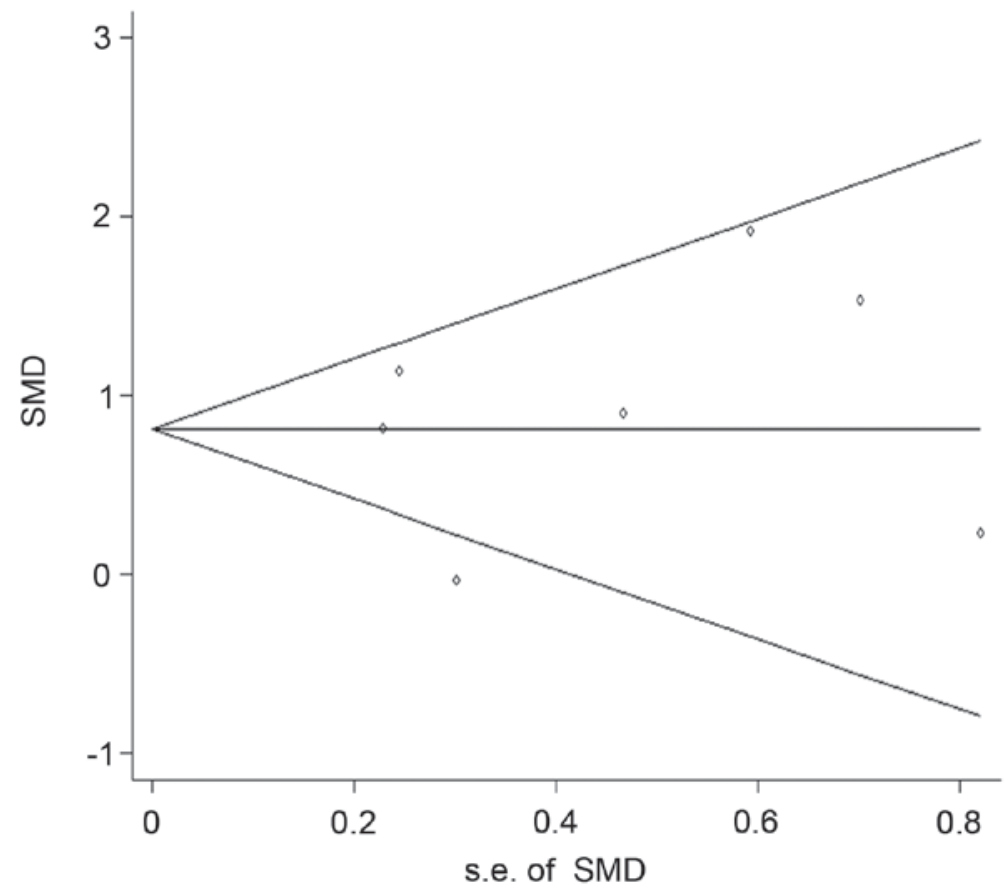

Figure 1. Forest plot and Begg's funnel plot of miR-9-3p expression in microarrays from GEO database. (A) Forest plot evaluating differences in miR-9-3p expression between lung adenocarcinoma tissues and normal lung tissues. Experimental group, high miR-9-3p expression; control group, low miR-9-3p expression. (B) Funnel plot for publication bias test of GEO microarrays. CI, confidence interval; GEO, Gene Expression Omnibus; miR, microRNA; s.e., standard error; SMD, standard mean difference.

differentially expressed in LINC00968-overexpressing A549 cells were profiled using miRNA microarray analysis. A total of five differentially expressed miRNAs were identified: One miRNA (miR-3675-3p) was upregulated and four miRNAs (miR-9-3p, miR-22-5p, miR-668-3p and miR-4536-3p) were downregulated in LINC00968-overexpressing A549 cells.
miR-9-3p $(\mathrm{FC}=0.569, \mathrm{P}=0.044)$ was selected for further analysis.

Confirmation of miR-9-3p downregulation in the GEO database. A meta-analysis was performed to verify miR-9-3p expression in other lung adenocarcinoma tissue microarrays. 
Table I. Significant GO terms most strongly enriched by target genes of miR-9-3p.

\begin{tabular}{lrrr}
\hline GO term & Count & P-value & FDR \\
\hline Biological processes & & & \\
GO:0045944: Positive regulation of transcription from RNA polymerase II promoter & 175 & $1.90 \times 10^{-11}$ & $3.62 \times 10^{-8}$ \\
GO:0045893: Positive regulation of transcription, DNA-templated & 97 & $6.97 \times 10^{-8}$ & $1.33 \times 10^{-4}$ \\
GO:0000122: Negative regulation of transcription from RNA polymerase II promoter & 123 & $3.09 \times 10^{-7}$ & $5.87 \times 10^{-4}$ \\
GO:0006366: Transcription from RNA polymerase II promoter & 93 & $7.81 \times 10^{-7}$ & $1.48 \times 10^{-3}$ \\
GO:0006351: Transcription, DNA-templated & 277 & $1.03 \times 10^{-6}$ & $1.95 \times 10^{-3}$ \\
Cellular components & & & \\
GO:0005737: Cytoplasm & 690 & $3.07 \times 10^{-13}$ & $4.72 \times 10^{-10}$ \\
GO:0005654: Nucleoplasm & 400 & $6.87 \times 10^{-12}$ & $1.05 \times 10^{-8}$ \\
GO:0005634: Nucleus & 703 & $7.26 \times 10^{-12}$ & $1.11 \times 10^{-8}$ \\
GO:0005923: Bicellular tight junction & 29 & $1.26 \times 10^{-5}$ & $1.93 \times 10^{-2}$ \\
GO:0016020: Membrane & 291 & $1.78 \times 10^{-5}$ & $2.73 \times 10^{-2}$ \\
Molecular function & & & \\
GO:0005515: Protein binding & 1133 & $1.65 \times 10^{-21}$ & $2.72 \times 10^{-18}$ \\
GO:0003730: mRNA 3'-UTR binding & 22 & $8.11 \times 10^{-9}$ & $1.34 \times 10^{-5}$ \\
GO:0043565: Sequence-specific DNA binding & 97 & $5.74 \times 10^{-8}$ & $9.48 \times 10^{-5}$ \\
GO:0003700: Transcription factor activity, sequence-specific DNA binding & 156 & $1.03 \times 10^{-7}$ & $1.71 \times 10^{-4}$ \\
GO:0001077: Transcriptional activator activity, RNA polymerase II core promoter & 54 & $1.36 \times 10^{-7}$ & $2.25 \times 10^{-4}$ \\
proximal region sequence-specific binding & & &
\end{tabular}

FDR, false discovery rate; GO, Gene Ontology; miR, microRNA; UTR, untranslated region.

A total of seven eligible microarrays were identified. As significant heterogeneity was detected among the microarrays $\left(\mathrm{I}^{2}=59.3 \%, \mathrm{P}<0.05\right)$, the random-effects model was used to assess the pooled SMD and 95\% CIs. Pooled SMD was 0.86 (95\% CI, 0.40-1.32, $\mathrm{P}=0.022$; Fig. 1A), which suggested that the expression of miR-9-3p in lung adenocarcinoma tissues was higher compared with normal lung tissues. Funnel plots revealed no significant publication bias in the meta-analysis (Begg's test, $\mathrm{P}=0.681$; Egger's test: $\mathrm{P}=0.681$; Fig. 1B).

Functional enrichment analysis of miR-9-3p target genes. A total of nine miRNA target prediction algorithms were used to identify target genes of miR-9-3p. Of the 7856 predicted target genes, 2047 genes overlapped in at least three datasets. To elucidate the biological function of miR-9-3p, functional enrichment analysis was implemented using these 2,047 target genes. Positive regulation of transcription from RNA polymerase II promoter (GO:0045944) was the most significant biological process term. Cytoplasm (GO:0005737) was the most significant cellular component term, and protein binding (GO:0005515) was the most significant molecular function term (Table I). A schematic of the top five most significant GO processes are presented in Fig. 2A.

The biological relevance of KEGG pathways that were enriched in these target genes was analyzed. The targets were involved in many cancer-associated pathways (Table II). The top five KEGG pathways that were enriched in the target genes are listed in Fig. 2B. KEGG pathway analysis identified many important signaling pathways [e.g., AMP-activated protein kinase (AMPK) and forkhead box O1 signaling pathways] known to be involved in the development, invasion, and metastasis of cancer. These findings indicate the biological relevance of miR-9-3p.

PPI network. To understand the interactions between target genes and their associations with LINC00968 and miR-9-3p, the predicted genes were integrated with the correlated genes. A total of 6,515 and 2,677 genes were correlated with LINC00968 and miR-9-3p, respectively, from the TCGA database. Using a Venn diagram, the 2,047 predicted target genes were integrated with the 6515 LINC00968-associated genes and the 2677 miR-9-3p-associated genes, uncovering 120 objective genes. To depict the complex PPI relationships among these objective genes, the PPI network of the top 30 genes were visualized by STRING (Fig. 3A). The number of links between genes was calculated, and a list of the top 30 most connected genes was obtained. Among them, cyclin A2 (CCNA2) had the greatest connectivity (Fig. 3B) and was subjected to further validation.

Validation of regulatory axis of LINC00968 in TCGA database. Predictive performances of LINC00968, miR-9-3p and CCNA2 in lung adenocarcinoma were estimated by ROC curve analysis. Areas under the ROC curve (AUCs) of components in this regulatory axis among lung adenocarcinoma patients from TCGA database were as follows: LINC00968, AUC $=0.988$ (95\% CI, 0.977-0.998, $\mathrm{P}=0.000)$, miR-9-1, AUC $=0.983$ (95\% CI, 0.967-0.999, P=0.000), miR-9-2 AUC $=0.982(95 \%$ CI, 0.968-0.997, $\mathrm{P}=0.000)$, miR-9-3 AUC $=0.981(95 \% \mathrm{CI}$, 0.968-0.995, $\mathrm{P}=0.000)$, and CCNA2, AUC $=0.960$ (95\% CI, 
A

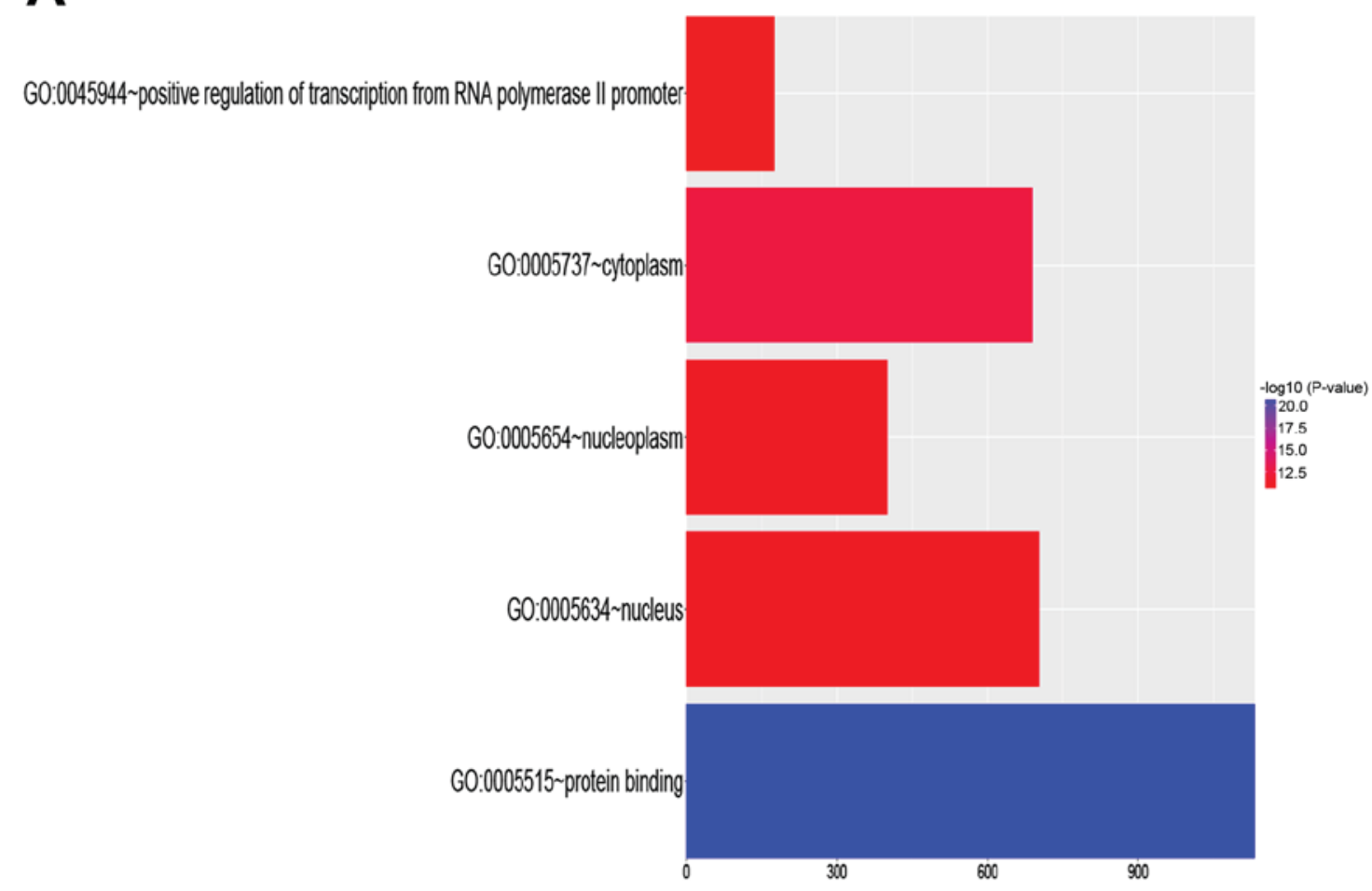

B

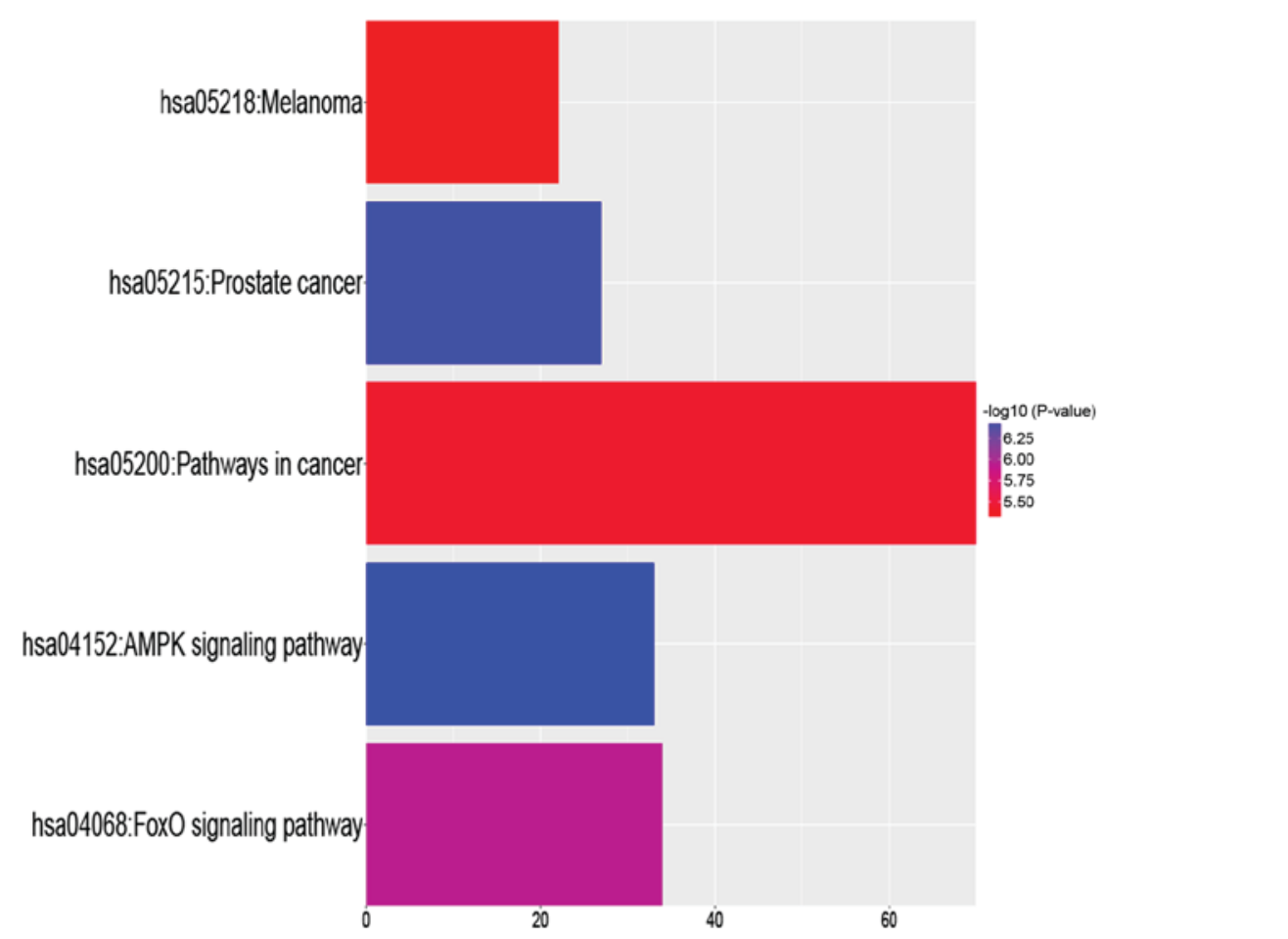

Figure 2. Results of functional enrichment analysis. (A) Top five most enriched Gene Ontology terms. (B) Top five most enriched Kyoto Encyclopedia of Genes and Genomes pathways. Blue, high significance; red, low significance; AMPK, AMP-activated protein kinase; FoxO, forkhead box O1.

0.921-0.999, $\mathrm{P}=0.000)$. The data suggested that the three signatures had good predictive performance in lung adenocarcinoma patients.

A Kaplan-Meier survival analysis was performed to determine the effects of the three components on predicting clinical outcome (Fig. 4). The median gene expression (which, for LINC00968, CCNA2, miR-9-1, miR-9-2 and miR-9-3 were $20.70,694.90,1009.58,986.66$ and 1010.02 , respectively) was used to separate the patients into high and low expression groups in TCGA datasets. The number of patients expressing LINC00968 and CCNA2 in the high-expression and low-expression groups in the present study was 267 . The number of patients expressing miR-9-3p in the high-expression and low-expression groups was 260. Low expression of LINC00968 (Fig. 4A) or high expression of CCNA2 (Fig. 4E) was a significant predictor of poor prognosis for overall 
Table II. Kyoto Encyclopedia of Genes and Genomes pathways most strongly enriched by target genes of miR-9-3p.

\begin{tabular}{llll}
\hline Terms & Count & P-value & FDR \\
\hline hsa04152: AMPK signaling pathway & 33 & $3.61 \times 10^{-7}$ & $4.74 \times 10^{-4}$ \\
hsa05215: Prostate cancer & 27 & $3.74 \times 10^{-7}$ & $4.91 \times 10^{-4}$ \\
hsa04068: FoxO signaling pathway & 34 & $1.14 \times 10^{-6}$ & $3.49 \times 10^{-3}$ \\
hsa05200: Pathways in cancer & 70 & $3.77 \times 10^{-6}$ & $4.95 \times 10^{-3}$ \\
hsa05218: Melanoma & 22 & $6.87 \times 10^{-6}$ & $6.39 \times 10^{-3}$ \\
hsa04390: Hippo signaling pathway & 35 & $2.45 \times 10^{-6}$ & $8.91 \times 10^{-3}$ \\
hsa04550: Signaling pathways regulating & 32 & & $3.22 \times 10^{-2}$ \\
pluripotency of stem cells & & $2.10 \times 10^{-4}$ \\
hsa05214: Glioma & 18 & $2.70 \times 10^{-4}$ \\
hsa03015: mRNA surveillance pathway & 22 & $2.87 \times 10^{-4}$ \\
hsa04151: PI3K-Akt signaling pathway & 57 & $2.75 \times 10^{-1}$ \\
\hline
\end{tabular}

AMPK, AMP-activated protein kinase, catalytic, $\alpha-1$; FDR, false discovery rate; FoxO, forkhead box O1; miR, microRNA; PI3K, phosphoinositide-3-kinase.

survival in lung adenocarcinoma patients. These findings suggested that LINC00968 and CCNA2 had good potential as diagnostic and prognostic indicators.

Finally, the underlying mechanisms of the LINC00968 regulatory axis in lung adenocarcinoma were investigated. In our previous GEO results, miR-9-3p was detected to be significantly upregulated in tumor tissues and had oncogenic potential in lung adenocarcinoma. Therefore, the correlation between LINC00968 and miR-9-3p (including miR-9-1, miR-9-2, and miR-9-3 in TCGA database) was examined. Negative correlations were identified between LINC00968 and miR-9-3p (Fig. 5A-C) and between LINC00968 and CCNA2 (Fig. 5D), whereas a positive correlation between miR-9-3p and CCNA2 (Fig. 5E-G) was identified in patients with lung adenocarcinoma. Therefore, it is concluded that the regulatory role of LINC00968 in lung adenocarcinoma might involve the LINC00968/miR-9-3p/CCNA2 axis.

\section{Discussion}

In recent years, microarray studies, RNA sequencing, and quantitative reverse-transcription PCR have been used to investigate the differential expression profiles of lncRNAs in various types of cancer. LncRNAs regulate diverse biological processes and act as oncogenes or tumor suppressors in many cancer types (24-28). As such, lncRNAs have great potential for use as molecular biomarkers in cancer diagnosis and prognosis. For example, the lncRNA, metastasis associated in lung adenocarcinoma transcript 1 , was found to promote the proliferation and metastasis of lung cancer (29). The authors previously reported that the IncRNA LINC00968 was differentially expressed in normal vs. lung tumor tissues in the GEO database (16). However, the regulatory mechanisms of LINC00968 in lung cancer have been unclear.

LncRNAs and miRNAs may have important interactions that contribute to cancer development. Evidence for this ceRNA hypothesis has been provided by several reports. For instance, lncRNA cancer susceptibility candidate 2 (CASC2) was shown to inhibit cell proliferation and tumor growth by regulating miR-18a in colorectal cancer (30). C032469 regulated telomerase reverse transcriptase expression by binding to miR-1207-5p in gastric cancer (31). Growth Arrest Specific Transcript 5 suppressed carcinogenesis in NSCLC by repressing miR-135b expression (32). In light of this research, the present authors postulated that LIN00968 may act as a ceRNA in NSCLC. The expression profile of miR-9-3p in NSCLC A549 cells that overexpressed LINC00968 was detected. Finally, bioinformatic analysis was used to reveal the potential regulatory role of LINC00968 in lung adenocarcinoma.

miR-9-3p has been demonstrated to regulate biological functions in multiple types of cancer. For example, Yang et al (33) reported that $\mathrm{H}_{2} \mathrm{O}_{2}$-induced apoptosis of glioma cells was facilitated by miR-9-3p through downregulation of Herpud1 expression. In another study, miR-9-3p inhibited cell proliferation by targeting the PDZ-binding motif TAZ in hepatocellular carcinoma (34). miR-9-3p was detected to suppress tumor biological behaviors in gastric cancer (35). Other studies have suggested regulatory roles for miR-9-3p in tumor initiation, growth and progression in NSCLC (36-38).

Results of the present analysis of miR-9-3p target genes indicated that the most enriched GO term was protein binding, a key process in carcinogenesis. For example, adiponectin was detected to inhibit proliferation of A549 cells through its downregulation of binding to cyclic AMP response element (39). Another study described a fusion protein that could be used to improve antitumor activities in NSCLC by targeting epidermal growth factor receptor (EGFR) and insulin-like growth factor 1 receptor (IGFR1) (40). In the present study, KEGG pathway analysis indicated that the most enriched pathway was the AMPK signaling pathway. Phosphoinositide-3-kinase/Akt/mechanistic target of rapamycin kinase and Raf/Mek/extracellular signal-regulated kinase, which are regulated by AMPK, are crucial signaling pathways related to cell growth, survival and molecular expression (41). Furthermore, numerous studies have demonstrated that AMPK is an important mediator of NSCLC progression (42-44). Taken together, 
A

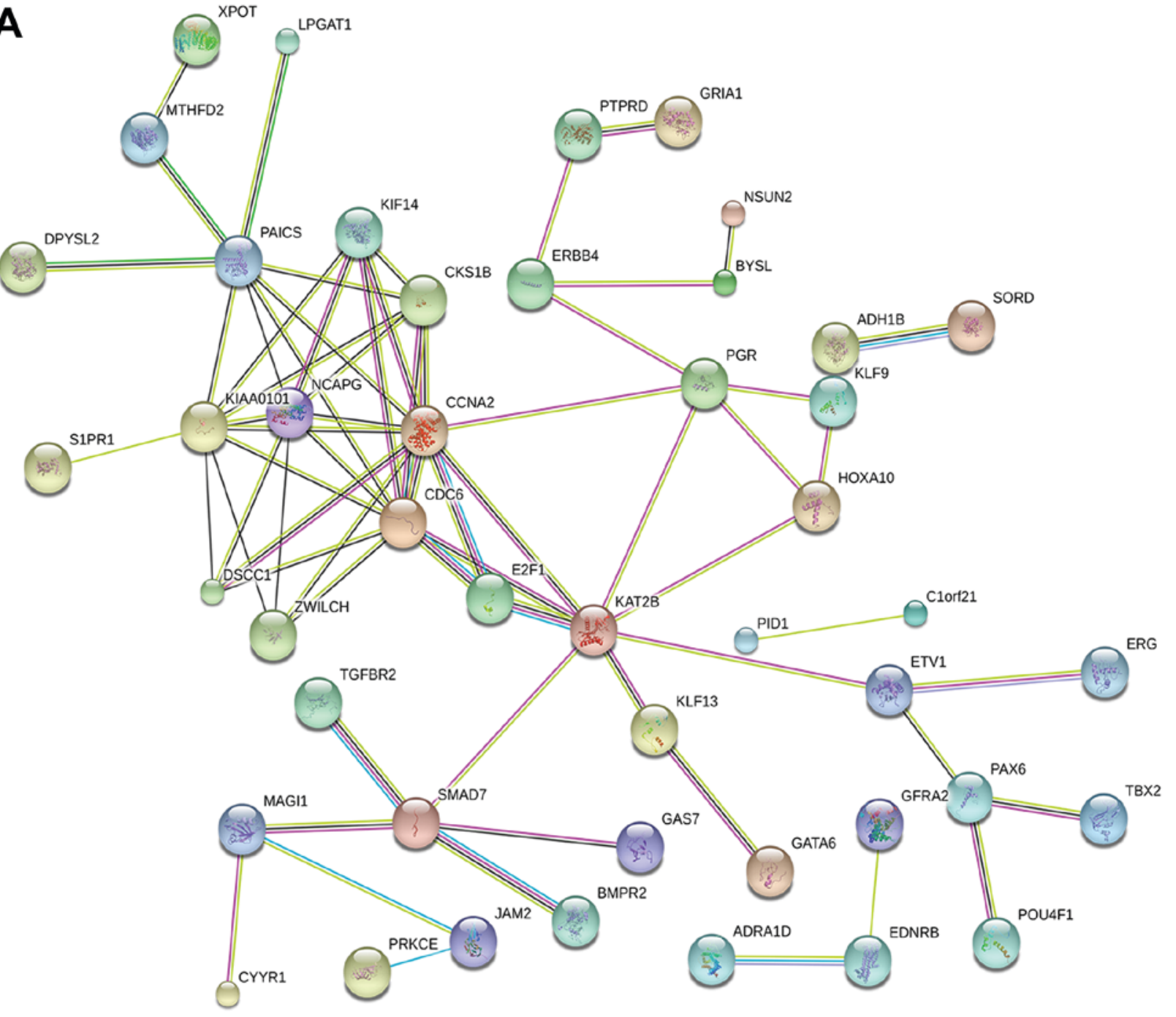

B

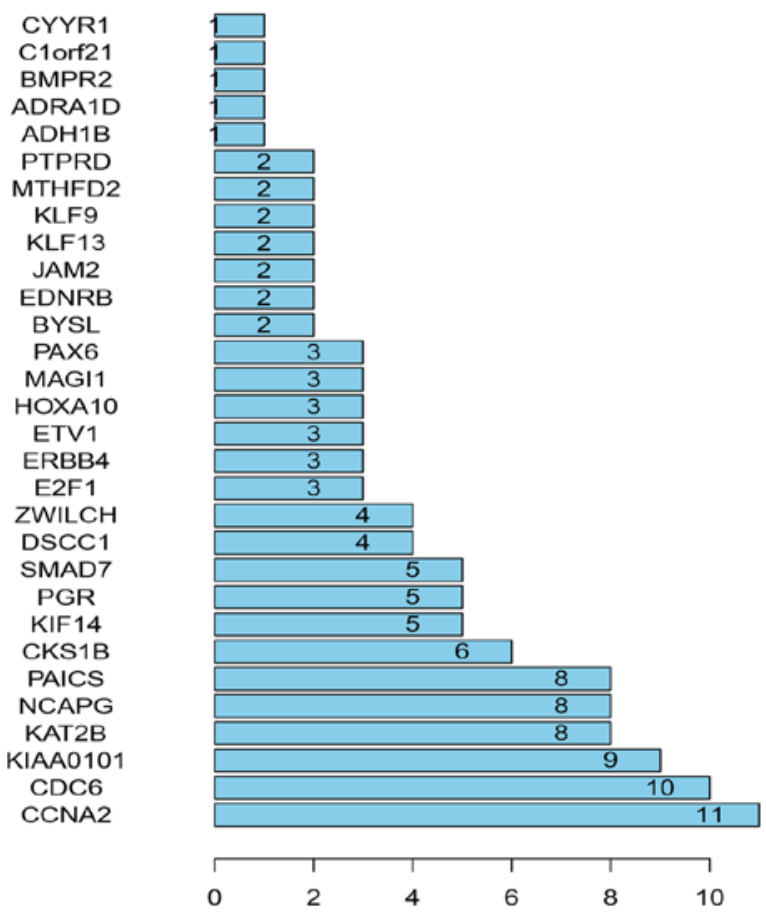

Figure 3. Analysis of functional protein association. (A) PPI network of 120 objective genes. (B) Top 30 most connected genes of PPI that are arranged by connectivity. PPI, Protein-protein interaction. 
A

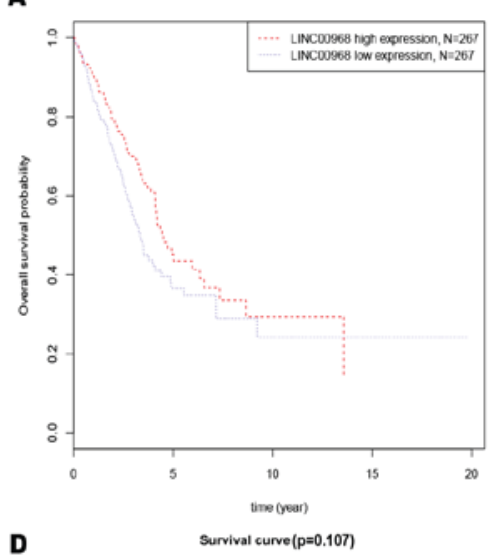

D

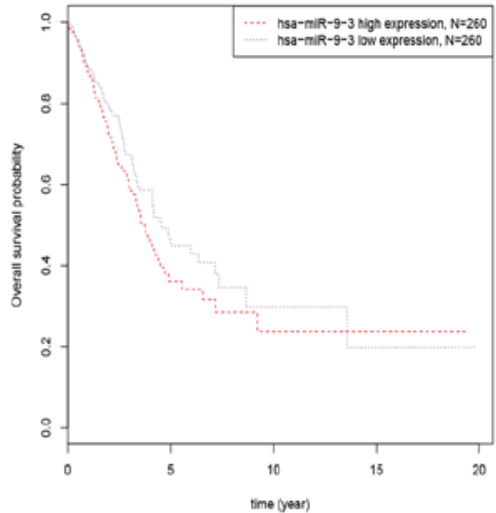

B

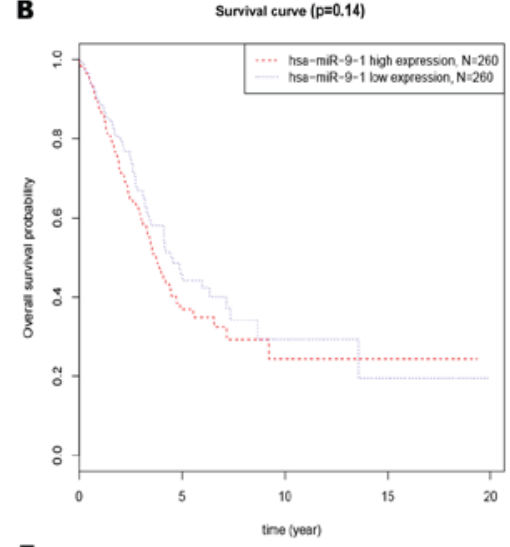

E

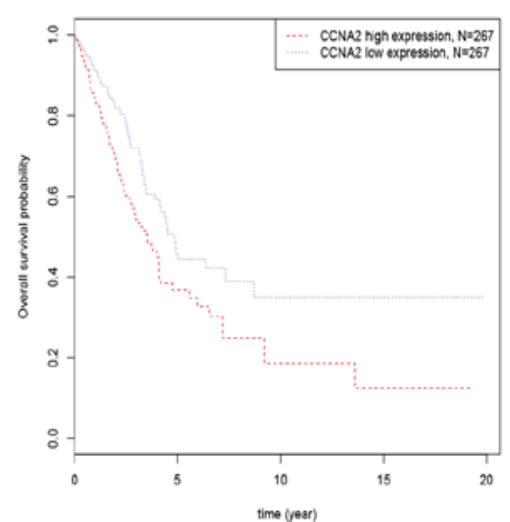

c

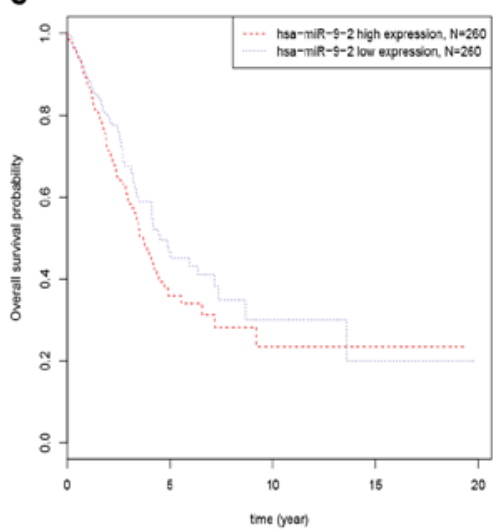

Figure 4. Overall survival analysis of LINC00968, miR-9-3p and CCNA2 expression in lung adenocarcinoma from The Cancer Genome Atlas database. Survival curves for (A) LINC00968, (B) miR-9-1, (C) miR-9-2, (D) miR-9-3 and (E) CCNA2. Red and green lines indicate high and low levels of expression, respectively. X-axis, overall survival time (year). y-axis, overall survival probability. The number of patients expressing LINC00968 and CCNA2 in the highexpression and low-expression groups is 267 . The number of patients expressing miR-9-3p in the high-expression and low-expression groups is 260 . CCNA2, cyclin A2; miR, microRNA.

all of these results indicate that miR-9-3p participates in the tumorigenesis process and acts as an efficient regulator of NSCLC development.

As an important regulator of the cell cycle and cell proliferation, CCNA2 has been consistently demonstrated to be closely associated with the pathogenesis of lung cancer. For instance, coiled-coil domain containing 106 promoted proliferation of A549 and H1299 cells by upregulating CCNA2 expression (45). In another report, miR-137 and miR-30a inhibited tumor growth by inducing cell-cycle arrest and downregulating cell cycle-associated regulators, including CCNA2 $(46,47)$. In addition, the gene E2F1 is an important transcription factor and is one of the most important pro-metastatic genes. Mei et al (48) observed that E2F1 had a significant effect on the prognosis of patients and was closely associated with patient survival, suggesting that E2F1 has an important role in the progression of clear cell renal cell carcinoma and hepatocellular carcinoma.

In the present study, the correlation analysis results suggested the existence of tight connections among LINC00968, miR-9-3p and CCNA2 in lung adenocarcinoma. Additionally, results of the Kaplan-Meier survival analysis indicated that LINC00968 may be a prospective biomarker for the diagnosis and prognosis of lung adenocarcinoma. Overall, these findings indicate that the LINC00968/miR-9-3p/CCNA2 regulatory axis deserves further research and investigation. The continuous development of novel research tools for identifying signal transduction pathways and discovering new therapeutic targets was inspired by the current investigation of molecular tumor profiles. Using tools to identify the prognostic and diagnostic markers in the present study presents an analysis concept based on existing tools, a number of which are available online, including $\mathrm{R}$ packages, Cytoscape, GO and the KEGG (49). Furthermore, the combination of the application of these tools and experimental verification will have a more important role in the identification of miRNAs. Therefore, the regulatory mechanisms of LINC00968 should be experimentally verified.

In conclusion, LINC00968 might have important functions in NSCLC via the LINC00968/miR-9-3p/CCNA2 regulatory axis. Results of the present study describe a novel approach to research the underlying mechanisms of LINC00968 in the tumorigenesis and development of NSCLC.

\section{Acknowledgements}

Not applicable.

\section{Funding}

The present study was supported by Guangxi Key Project of Science and Technology (grant no. 1598012-30), the Fund of the Guangxi Provincial Health Bureau Scientific 
A

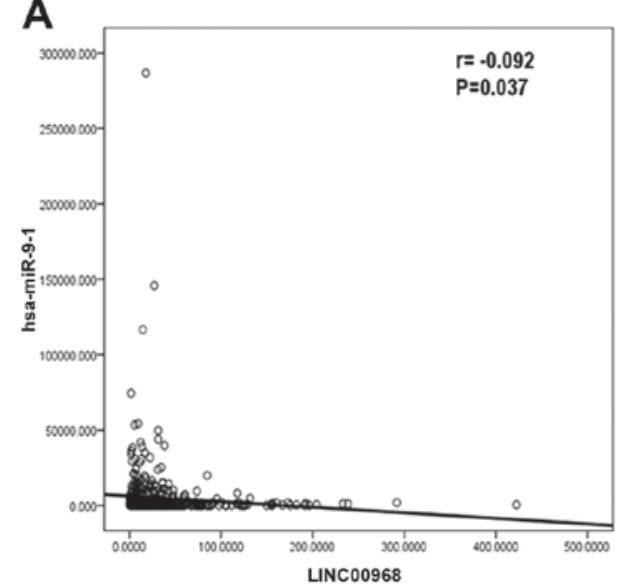

C

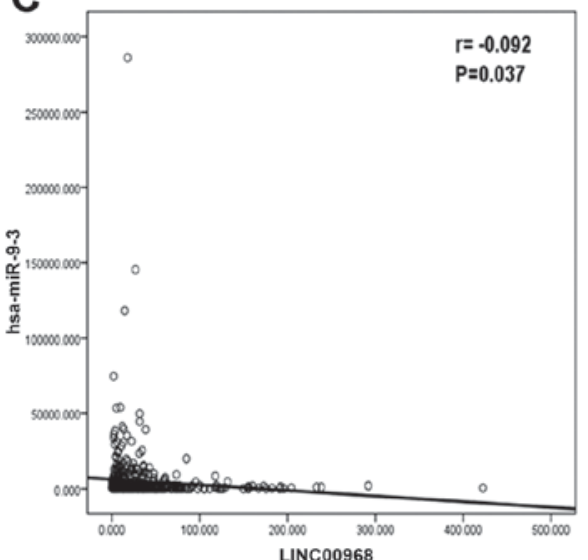

E

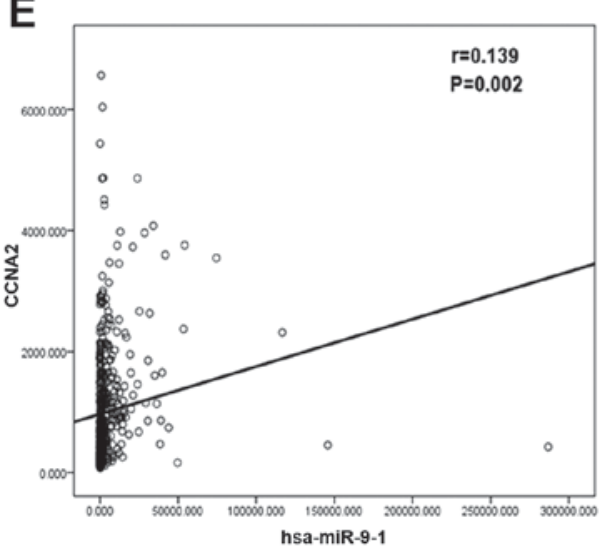

B

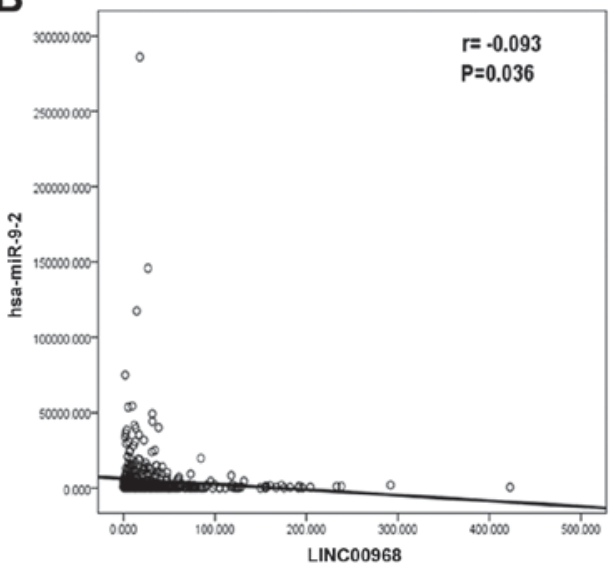

D

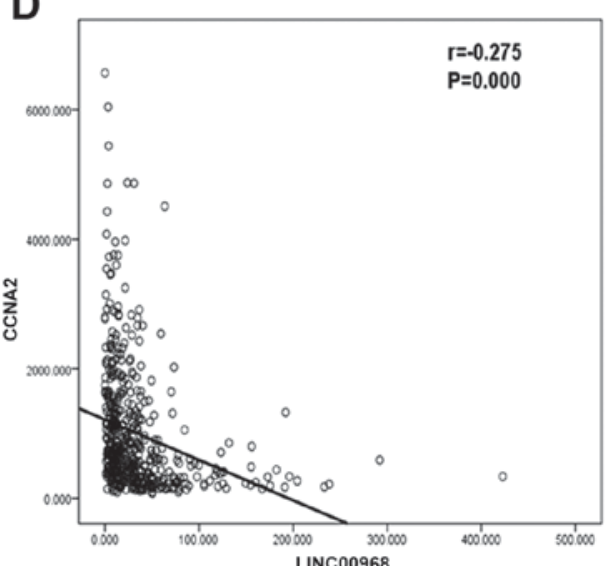

F

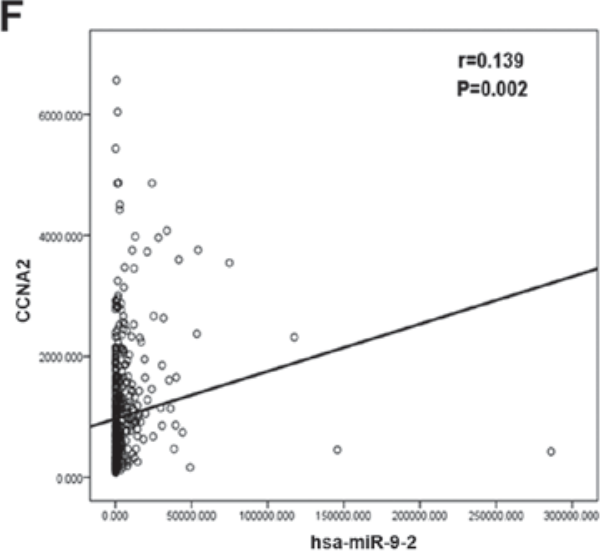

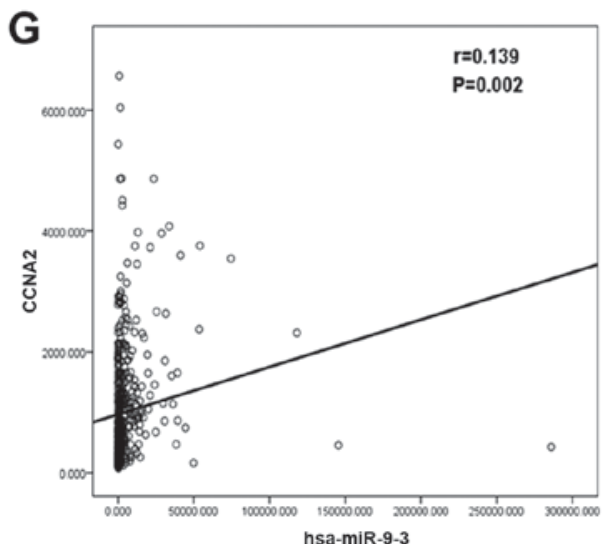

Figure 5. Correlation analysis among LINC00968, miR-9-3p, and CCNA2 expression in lung adenocarcinoma based on TCGA database. Correlations (A) between LINC00968 and miR-9-1, (B) between LINC00968 and miR-9-2, (C) between LINC00968 and miR-9-3, (D) between LINC00968 and CCNA2, (E) between miR-9-1 and CCNA2, (F) between miR-9-2 and CCNA2, (G) between miR-9-3 and CCNA2. CCNA2, cyclin A2; miR, microRNA; TCGA, The Cancer Genome Atlas. 
Research Project (grant no. Z2013201), the Fund of the National Natural Science Foundation of China (grant nos. NSFC81660488, NSFC81360327 and NSFC81560469), and the Natural Science Foundation of Guangxi, China (grant no. 2015GXNSFCA139009). The funders had no role in the study design, the data collection and analysis, the decision to publish, or the preparation of the manuscript.

\section{Availability of data and materials}

The datasets generated and analyzed during the current study are available in GEO (https://www.ncbi.nlm.nih.gov/gds) and TCGA (http://cancergenome.nih.gov/).

\section{Authors' contributions}

SKL and GC conceived the idea for the study. DYL, WJC and JS analyzed and interpreted the data. DYL was a major contributor in writing the manuscript. All authors read and approved the final manuscript.

\section{Ethics approval and consent to participate}

Not applicable.

\section{Consent for publication}

Not applicable.

\section{Competing interests}

The authors declare that there are no competing interests.

\section{References}

1. Siegel RL, Miller KD and Jemal A: Cancer statistics, 2017. CA Cancer J Clin 67: 7-30, 2017.

2. Travis WD: The 2015 WHO classification of lung tumors. Pathologe 35 (Suppl 2): S188, 2014.

3. Yang R, Li P, Zhang G, Lu C, Wang H and Zhao G: Long non-coding RNA XLOC_008466 functions as an oncogene in human non-small cell lung cancer by targeting miR-874. Cell Physiol Biochem 42: 126-136, 2017.

4. He Y, Meng XM, Huang C, Wu BM, Zhang L, Lv XW and Li J: Long noncoding RNAs: Novel insights into hepatocelluar carcinoma. Cancer Lett 344: 20-27, 2014.

5. Wan L, Sun M, Liu GJ, Wei CC, Zhang EB, Kong R, Xu TP, Huang MD and Wang ZX: Long noncoding RNA PVT1 promotes non-small cell lung cancer cell proliferation through epigenetically regulating LATS2 expression. Mol Cancer Ther 15: 1082-1094, 2016.

6. Nie W, Ge HJ, Yang XQ, Sun X, Huang H, Tao X, Chen WS and Li B: LncRNA-UCA1 exerts oncogenic functions in non-small cell lung cancer by targeting miR-193a-3p. Cancer Lett 371: 99-106, 2016.

7. Liz $\mathrm{J}$ and Esteller M: lncRNAs and microRNAs with a role in cancer development. Biochim Biophys Acta 1859: 169-176, 2016

8. Liang WC, Fu WM, Wong CW, Wang Y, Wang WM, Hu GX, Zhang L, Xiao LJ, Wan DC, Zhang JF and Waye MM: The lncRNA H19 promotes epithelial to mesenchymal transition by functioning as miRNA sponges in colorectal cancer. Oncotarget 6: 22513-22525, 2015.

9. Tay Y, Rinn J and Pandolfi PP: The multilayered complexity of ceRNA crosstalk and competition. Nature 505: 344-352, 2014.

10. Arima C, Kajino T, Tamada Y, Imoto S, Shimada Y, Nakatochi M, Suzuki M, Isomura H, Yatabe Y, Yamaguchi T, et al: Lung adenocarcinoma subtypes definable by lung development-related miRNA expression profiles in association with clinicopathologic features. Carcinogenesis 35: 2224-2231, 2014.
11. Fujita Y, Yagishita S, Hagiwara K, Yoshioka Y, Kosaka N, Takeshita F, Fujiwara T, Tsuta K, Nokihara H, Tamura T, et al: The clinical relevance of the miR-197/CKS1B/STAT3-mediated PD-L1 network in chemoresistant non-small-cell lung cancer. Mol Ther 23: 717-727, 2015.

12. Xia T, Liao Q, Jiang X, Shao Y, Xiao B, Xi Y and Guo J: Long noncoding RNA associated-competing endogenous RNAs in gastric cancer. Sci Rep 4: 6088, 2014.

13. Zhang J, Fan D, Jian Z, Chen GG and Lai PB: Cancer specific long noncoding RNAs show differential expression patterns and competing endogenous RNA potential in hepatocellular carcinoma. PLoS One 10: e0141042, 2015.

14. Zhou X, Liu J and Wang W: Construction and investigation of breast-cancer-specific ceRNA network based on the mRNA and miRNA expression data. IET Syst Biol 8: 96-103, 2014.

15. Zhou M, Diao Z, Yue X, Chen Y, Zhao H, Cheng L and Sun J: Construction and analysis of dysregulated lncRNA-associated ceRNA network identified novel lncRNA biomarkers for early diagnosis of human pancreatic cancer. Oncotarget 7 : 56383-56394, 2016.

16. Yang J, Lin J, Liu T, Chen T, Pan S, Huang W and Li S: Analysis of lncRNA expression profiles in non-small cell lung cancers (NSCLC) and their clinical subtypes. Lung Cancer 85: 110-115, 2014.

17. Xu X, Wang X, Fu B, Meng L and Lang B: Differentially expressed genes and microRNAs in bladder carcinoma cell line 5637 and T24 detected by RNA sequencing. Int J Clin Exp Pathol 8: 12678-12687, 2015.

18. Li Q, Ge X, Xu X, Zhong Y and Qie Z: Comparison of the gene expression profiles between gallstones and gallbladder polyps. Int J Clin Exp Pathol 7: 8016-8023, 2014.

19. Jiang CM, Wang XH, Shu J, Yang WX, Fu P, Zhuang LL and Zhou GP: Analysis of differentially expressed genes based on microarray data of glioma. Int J Clin Exp Med 8: 17321-17332, 2015.

20. Chen L, Zhuo D, Chen J and Yuan H: Screening feature genes of lung carcinoma with DNA microarray analysis. Int J Clin Exp Med 8: 12161-12171, 2015.

21. Huang da W, Sherman BT and Lempicki RA: Systematic and integrative analysis of large gene lists using DAVID bioinformatics resources. Nat Protoc 4: 44-57, 2009.

22. Szklarczyk D, Franceschini A, Wyder S, Forslund K, Heller D, Huerta-Cepas J, Simonovic M, Roth A, Santos A, Tsafou KP, et al: STRING v10: Protein-protein interaction networks, integrated over the tree of life. Nucleic Acids Res 43 (Database Issue): D447-D452, 2015.

23. R Core Team: R: A language and environment for statistical computing, $R$ foundation for statistical computing, Vienna, Austria, 2013. https://www.R-project.org.

24. Naemura M, Murasaki C, Inoue Y, Okamoto H and Kotake Y: Long noncoding RNA ANRIL regulates proliferation of non-small cell lung cancer and cervical cancer cells. Anticancer Res 35: 5377-5382, 2015.

25. Liu YY, Chen ZH, Peng JJ, Wu JL, Yuan YJ, Zhai ET, Cai SR, He YL and Song W: Up-regulation of long non-coding RNA XLOC_010235 regulates epithelial-to-mesenchymal transition to promote metastasis by associating with Snaill in gastric cancer. Sci Rep 7: 2461, 2017.

26. Hua F, Li CH, Chen XG and Liu XP: Long noncoding RNA CCAT2 knockdown suppresses tumorous progression by sponging miR-424 in epithelial ovarian cancer. Oncol Res 26: 241-247, 2018.

27. Meng Q, Ren M, Li Y and Song X: LncRNA-RMRP acts as an oncogene in lung cancer. PLoS One 11: e0164845, 2016.

28. Cao S, Wang Y, Li J, Lv M, Niu H and Tian Y: Tumor-suppressive function of long noncoding RNA MALAT1 in glioma cells by suppressing miR-155 expression and activating FBXW7 function. Am J Cancer Res 6: 2561-2574, 2016.

29. Chou J, Wang B, Zheng T, Li X, Zheng L, Hu J, Zhang Y, Xing Y and Xi T: MALAT1 induced migration and invasion of human breast cancer cells by competitively binding miR-1 with cdc 42 . Biochem Biophys Res Commun 472: 262-269, 2016.

30. Huang G, Wu X, Li S, Xu X, Zhu H and Chen X: The long noncoding RNA CASC2 functions as a competing endogenous RNA by sponging miR-18a in colorectal cancer. Sci Rep 6: 26524, 2016.

31. Zhang CG, Yin DD, Sun SY and Han L: The use of IncRNA analysis for stratification management of prognostic risk in patients with NSCLC. Eur Rev Med Pharmacol Sci 21: 115-119, 2017.

32. Xue Y, Ni T, Jiang Y and Li Y: Long noncoding RNA GAS5 inhibits tumorigenesis and enhances radiosensitivity by suppressing miR-135b expression in non-small cell lung cancer. Oncol Res 25: 1305-1316, 2017. 
33. Yang L, Mu Y, Cui H, Liang Y and Su X: MiR-9-3p augments apoptosis induced by $\mathrm{H} 2 \mathrm{O} 2$ through down regulation of Herpud1 in glioma. PLoS One 12: e0174839, 2017.

34. Higashi T, Hayashi H, Ishimoto T, Takeyama H, Kaida T, Arima K, Taki K, Sakamoto K, Kuroki H, Okabe H, et al: miR-9-3p plays a tumour-suppressor role by targeting TAZ (WWTR1) in hepatocellular carcinoma cells. Br J Cancer 113: 252-258, 2015.

35. Meng Q, Xiang L, Fu J, Chu X, Wang C and Yan B: Transcriptome profiling reveals miR-9-3p as a novel tumor suppressor in gastric cancer. Oncotarget 8: 37321-37331, 2017.

36. Yuva-Aydemir Y,Simkin A, Gascon E and Gao FB: MicroRNA-9: Functional evolution of a conserved small regulatory RNA. RNA Biol 8: 557-564, 2011.

37. Chen X, Zhu L, Ma Z, Sun G, Luo X, Li M, Zhai S, Li P and Wang X: Oncogenic miR-9 is a target of erlotinib in NSCLCs. Sci Rep 5: 17031, 2015.

38. Mitra R, Edmonds MD, Sun J, Zhao M, Yu H, Eischen CM and Zhao Z: Reproducible combinatorial regulatory networks elucidate novel oncogenic microRNAs in non-small cell lung cancer. RNA 20: 1356-1368, 2014

39. Illiano M, Nigro E, Sapio L, Caiafa I, Spina A, Scudiero O, Bianco A, Esposito S, Mazzeo F, Pedone PV, et al: Adiponectin down-regulates CREB and inhibits proliferation of A549 lung cancer cells. Pulm Pharmacol Ther 45: 114-120, 2017.

40. Guo XF, Zhu XF, Cao HY, Zhong GS, Li L, Deng BG, Chen P, Wang PZ, Miao QF and Zhen YS: A bispecific enediyne-energized fusion protein targeting both epidermal growth factor receptor and insulin-like growth factor 1 receptor showing enhanced antitumor efficacy against non-small cell lung cancer. Oncotarget 8: 27286-27299, 2017.

41. Troncone $\mathrm{M}$, Cargnelli SM, Villani LA, Isfahanian $\mathrm{N}$ Broadfield LA, Zychla L, Wright J, Pond G, Steinberg GR and Tsakiridis T: Targeting metabolism and AMP-activated kinase with metformin to sensitize non-small cell lung cancer (NSCLC) to cytotoxic therapy: Translational biology and rationale for current clinical trials. Oncotarget 8: 57733-57754, 2017.
42. Yim NH, Hwang YH, Liang C and Ma JY: A platycoside-rich fraction from the root of Platycodon grandiflorum enhances cell death in A549 human lung carcinoma cells via mainly AMPK/mTOR/AKT signal-mediated autophagy induction. J Ethnopharmacol 194: 1060-1068, 2016.

43. Zhang Y, Li ZY, Hou XX, Wang X, Luo YH, Ying YP and Chen G: Clinical significance and effect of AEG-1 on the proliferation, invasion, and migration of NSCLC: A study based on immunohistochemistry, TCGA, bioinformatics, in vitro and in vivo verification. Oncotarget 8: 16531-16552, 2017.

44. Praveen P, Hülsmann H, Sültmann H, Kuner R and Fröhlich H Cross-talk between AMPK and EGFR dependent signaling in non-small cell lung cancer. Sci Rep 6: 27514, 2016.

45. Zhang X, Zheng Q, Wang C, Zhou H, Jiang G, Miao Y, Zhang Y, Liu Y, Li Q, Qiu X and Wang E: CCDC106 promotes non-small cell lung cancer cell proliferation. Oncotarget 8: 26662-26670, 2017.

46. Chen R, Zhang Y, Zhang C, Wu H and Yang S: miR-137 inhibits the proliferation of human non-small cell lung cancer cells by targeting SRC3. Oncol Lett 13: 3905-3911, 2017.

47. Wen XP, Ma HL, Zhao LY, Zhang W and Dang CX: MiR-30a suppresses non-small cell lung cancer progression through AKT signaling pathway by targeting IGF1R. Cell Mol Biol (Noisy-le-grand) 61: 78-85, 2015.

48. Mei Y, Yang JP and Qian CN: For robust big data analyses: A collection of 150 important pro-metastatic genes. Chin J Cancer 36: 16, 2017.

49. Van Laere S, Dirix L and Vermeulen P: Molecular profiles to biology and pathways: A systems biology approach. Chin J Cancer 35: 53, 2016. 